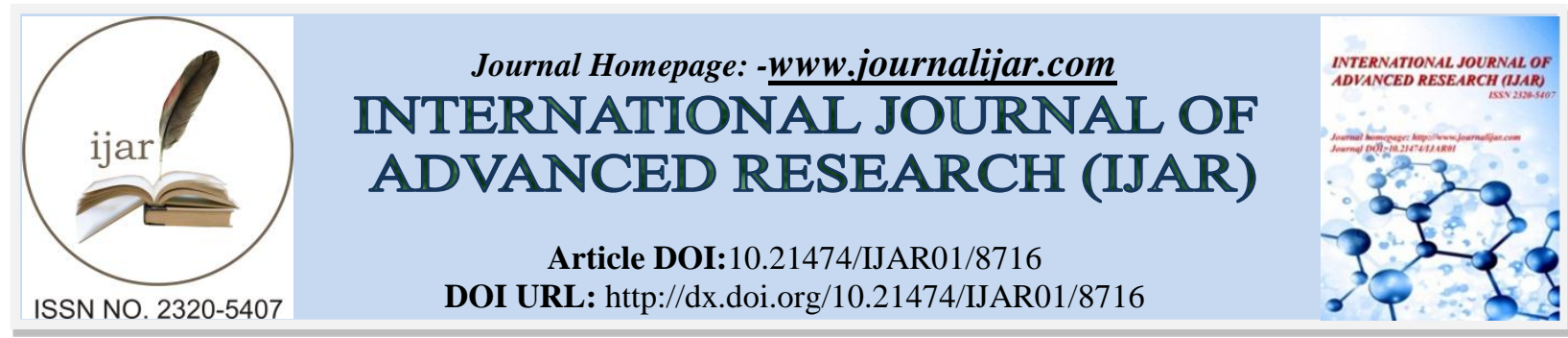

RESEARCH ARTICLE

\title{
COMPARATIVE STUDY ON WATER SORPTION AND SOLUBILITY OF THREE SOFT LINERS IN DIFFERENT SOLUTIONS: AN IN-VITRO STUDY.
}

Anagha Menon, Bhavana B.L, Rupesh P.L, Basavaraj S.Salagundi, Savitha K.C and Nithya. R. Krishnan.

\section{Manuscript Info}

Manuscript History

Received: 17 January 2019

Final Accepted: 19 February 2019

Published: March 2019
Abstract

Aim: Two silicone based soft denture lining materials (Mollosil, Molloplast-B), and an acrylic resin based soft denture liner (Viscogel) were selected for comparative evaluation of sorption and solubility in different solutions at different time intervals.

Methodology: In this study two silicone based soft denture lining materials (Mollosil, Molloplast-B), and acrylic based soft denture liner (Viscogel) were selected for comparative evaluation of sorption and solubility in different solutions at different time intervals. 50 specimens for each material: Mollosil, Molloplast-B and Viscogel were prepared using brass dies of dimensions - $50 \mathrm{~mm}$ diameter, $0.5 \mathrm{~mm}$ thickness. The specimens were stored in different solutions (distilled water, denture cleanser and artificial saliva- acidic, basic, neutral), and tested after 1,4 and 16 weeks for sorption and solubility. Analysis of variance was used to find the significant differences between the materials at all time intervals.

Result: The acrylic resin soft lining materials had higher solubility (3.920\% Viscogel in acidic saliva) and absorption (3.138\% Viscogel in distilled water) than Molloplast-B after 16 weeks of aging.

Conclusion: The silicone based soft liner Mollosil, showed the highest percentage of sorption and solubility in artificial saliva than in distilled water, while an acrylic based Viscogel showed the highest percentage solubility in artificial saliva, and lowest percentage of sorption in distilled with the exception of Molloplast-B, which showed the highest percentage of solubility and sorption in distilled water, compared to all these soft lining materials.

Copy Right, IJAR, 2019,. All rights reserved.

\section{Introduction:-}

Soft denture liners are indispensable in modern removable prosthodontics because of their role in restoring health to inflamed mucosa and distorted denture supporting tissues. ${ }^{1}$ They act as a cushion for the denture-bearing mucosa through absorption and redistribution of forces transmitted to the stress-bearing areas of the edentulous ridges. ${ }^{2}$ The soft lining material is designed to distribute functional and non-functional stresses more evenly due to the elastic behavior. ${ }^{3}$

Long term soft denture lining materials (LTSDL) are used to alleviate the trauma associated with the use of complete dentures. Despite their established clinical efficacy, the use of long term soft denture lining materials has 
been limited due to the unfavorable effects of the oral environment on some of their mechanical and performance characteristics. Commercially available soft lining materials include silicone-based long term soft denture linings (SLTSDLs), acrylic based long term soft denture linings (ALTSDLs). ${ }^{4}$

Currently polymethylmethacrylate (PMMA) resin is used less frequently since plasticizer molecules present in acrylic based long term soft denture liners can separate polymer chains which lowers the glass transition temperature.4 However such plasticizers are not bound within the resin; therefore, they leach out of the resin, which is the primary reason for the change in mechanical properties of acrylic based soft denture lining materials. ${ }^{5}$

The water sorption and solubility of soft denture liners are properties that occur in the moist environment are often overlooked leading to changes in the mechanical properties, discoloration, swelling, odor, and easier bacterial adhesion to the lining's surface. ${ }^{2,4,6}$ Therefore, an ideal long term soft denture material should neither absorb water nor contain soluble or leachable components. ${ }^{2}$ Several investigators have studied sorption, solubility, resiliency, tear strength, elongation, and bond strength of soft denture liners over a period of time. ${ }^{3,6,8}$ Some investigators have used varying experimental protocols, including different samples (thickness, diameter), aging times and aging solutions. ${ }^{4}$

Water sorption of long term soft denture lining material is dependent on the leachable components and on the hydrophilicity of the matrix. ${ }^{4}$ In acrylic based soft denture liners, the sorption of water is high because of leaching of plasticizers than those of silicone based denture liners. ${ }^{4}$ Soft lining materials are constantly bathed in saliva,

and when out of mouth, they are usually immersed in denture cleansers or water for storage. In these situations, there are two processes likely to take place. Water or saliva can be absorbed into material, and plasticizers or other constituents of soft lining material can be leached. Both processes are important in the effects they are

likely to have on the physical properties of the material and its dimensional stability. To predict the clinical behavior, both the amount of water absorbed and the amount of soluble material lost must be measured over a period which is comparable with the oral environment. ${ }^{9,10,11,12,13}$

The purpose of this study was to evaluate the effect of five different storage media on water sorption and solubility of two silicone based soft denture liners (Mollosil, Molloplast-B) and a plasticized acrylic resin (Viscogel) on the basis of water sorption and solubility.

\section{Materials \& Methods:-}

The study comprised of 3 groups of 50 specimens of each material of which Group 2 was divided into 3 sub groups of each material (Mollosil, Molloplast-B and Viscogel).

Groups were divided as follows:

Group 1: Distilled water

Group 2: Artificial saliva:

1. Acidic saliva

2. Basic saliva

3. Neutral saliva

\section{Group 3: Denture cleanser}

Mollosil, (DETAX, Germany), (chairside soft liner), is available in paste form which consists of base and catalyst pastes. The base and catalyst pastes were mixed in equal proportion for 30 seconds on a clean glass slab with a cement spatula. This mixture was then placed inside the brass die and pressed for $1 \mathrm{~min}$ and $30 \mathrm{sec}$. with a glass slab, so that it spreads uniformly. The excess was removed with a BP blade (no.12). Once the material was set (7 $\min$ ), and the initial weight was established with electronic precision balance.

Viscogel, (DENTS-PLY, Germany), a temporary soft denture liner is available as powder (Polyethyl methacryalte) and liquid (Ethanol, Phthalyl butyl glycolate, Dibutyl phthalate). $3 \mathrm{gm}$ of powder and $2.2 \mathrm{ml}$ of liquid was dispensed into a mixing vessel and mixed for $30 \mathrm{sec}$ (at room temperature). When the mixture reached a suitable consistency, it was then placed inside the brass die, on a clean dry glass slab and pressed for 2-3 min so that the material spreads uniformly. Then the material was removed from the brass die, the excess was removed with a BP blade (N0.12), and the initial weight was established with electronic precision balance. 
Molloplast-B, (DETAX, Germany), is a permanent soft liner. A wax spacer, was made inside the brass die, which was then invested with dental plaster, the flasks were placed into boiling water and de-waxed for 10-15min. Molloplast-B was taken with a clean plastic spatula from the jar and was packed it into the acrylic flask. After the flask was closed, it was placed in the clamp for polymerization. The flasks were then placed in cold water and heated up slowly to $100 \mathrm{oC}$ for 2 hours. It was then de-flasked after self cooling to room temperature. Then the initial weight was established with electronic precision balance.

The samples were then immersed in:

1. Solution 1: Distilled water

2. Solution 2: Neutral saliva

3. Solution 3: Acidic saliva

4. Solution 4: Basic saliva

5. Solution 5: Denture cleanser

The artificial saliva was of the following composition: $\mathrm{NaCl}: 0.400 \mathrm{gm} ; \mathrm{KCl}: 0.400 \mathrm{gm}$; CaCl2H2O:0.795gm; NaH2PO4:0.69gm; Na2S.9H2O:0.005gm; Urea 1.0gm; distilled water:100 ml. To obtain acidic, basic and neutral saliva the $\mathrm{pH}$ of artificial saliva was adjusted to 4, 7, 8 with $\mathrm{NaOH}$ or HCL and the volume was increased to $1 \mathrm{~L}$. Acidic saliva was made by adding a drop of HCL to artificial saliva, and the $\mathrm{pH}$ (4) was checked using a blue litmus paper which turned red. Basic saliva was made using $\mathrm{NaOH}$, and p $\mathrm{H}$ (7) was checked using a red litmus paper which turned blue. Neutral saliva, p H (8) there was no colour change in the litmus paper. The distilled water used in this study was commercially available Smart Water (Mumbai), denture cleanser was Fittydent (Mag. Hoeveler \& Co.Gmbh, Germany), and artificial saliva was (MP SAI ENTERPRISE Pvt. Ltd. Thane). The samples were tested in National Institute of Technology, Calicut.

Each specimen was dried in a desiccator containing anhydrous calcium chloride until a constant weight was obtained. This was considered to be the initial weight of the specimen(W1). The processed samples, 50 each for two silicones soft liner and 50 acrylic based soft liner were then placed in a sealed container each containing different solutions for 1,4 and 16 weeks at $37 \pm 2^{\circ} \mathrm{C}$ (Figure 7,8,9,10,11,12). After each time period, the samples were removed from their containers, excess water was removed by blotting with the filter paper, the samples were then weighed using electronic precision balance, capable of measuring $0.001 \mathrm{~g}$. This was the weight of the specimen after absorption (W2). The amount of soluble material lost was measured by placing the specimens back in the desiccator after each sorption cycle and then weighing them at regular intervals until a constant weight was obtained after desiccation (W3).

The above procedures were repeated, and sorption and solubility of data were collected for 1,4 and 16 weeks.

\section{Results:-}

Statistical analysis

1. The data was collected, coded and fed in SPSS (IBM Version 23) for statistical analysis. The descriptive statistics include mean and SD. Inferential statistics include ONE WAY ANOVA followed by POST HOC TUKEY'S TEST for multiple comparisons. The level of significance was set at $\mathrm{P}<0.05$ at $95 \%$ confidence interval.

2. Table 1 shows the mean solubility of Molloplast-B; after 1 week, in distilled water was $0.97400 \%$, acidic saliva was $0.56710 \%$, basic saliva was $0.40610 \%$, neutral saliva was $0.31160 \%$, and denture cleanser was $0.4910 \%$.

3. After 4 weeks, the mean solubility of Mollolplast-B, in distilled water was $1.05970 \%$, acidic saliva was $0.59600 \%$, basic saliva was $0.43230 \%$, neutral saliva was $0.34650 \%$, and denture cleanser was $0.5500 \%$.

4. After 16 weeks, the mean solubility of Mollolplast-B, in distilled water was $1.09646 \%$, acidic saliva was $0.62660 \%$, basic saliva was $0.46550 \%$, neutral saliva was $0.38310 \%$, and denture cleanser was $0.4580 \%$.

5. After 1week, 4weeks, and 16 weeks, Molloplast-B in denture cleanser showed the least solubility followed by neutral saliva, basic saliva, acidic saliva, and the highest solubility was seen in distilled water as depicted in Graph 1.Table 2 shows the mean sorption of Molloplast-B; after 1 week, in distilled water was $0.79430 \%$, acidic saliva was $0.60480 \%$, basic saliva was $0.38400 \%$, neutral saliva was $0.30730 \%$, and denture cleanser was $0.05500 \%$.

6. After 4 weeks, the mean sorption of Molloplast-B in distilled water was $0.83060 \%$, acidic saliva was $0.63420 \%$, basic saliva was $0.40770 \%$, neutral saliva was $0.33920 \%$, and denture cleanser was $0.06030 \%$. 
7. After16 weeks, the mean sorption of Molloplast-B in distilled water was $0.86960 \%$, acidic saliva was $0.66070 \%$, basic saliva was $0.41920 \%$, neutral saliva was $0.36080 \%$, and denture cleanser was $0.06030 \%$.

8. After 1, 4, and 16 weeks, Molloplast-B in denture cleanser showed the least sorption followed by neutral saliva, basic saliva and acidic saliva, while in distilled water Molloplast B showed the highest sorption as depicted in Graph 2.

9. Table 3 shows the mean sorption of Mollosil; after 1 week, in distilled water was $0.52850 \%$, acidic saliva was $0.97730 \%$, basic saliva was $0.32580 \%$, neutral saliva was $0.28940 \%$, and denture cleanser was $0.04900 \%$.

10. After 4 weeks, the mean sorption of Mollosil in distilled water was $0.58850 \%$, acidic saliva was $1.01250 \%$, basic saliva was $0.33720 \%$, neutral saliva was $0.31280 \%$, and denture cleanser was $0.05000 \%$.

11. After 16 weeks, the mean solubility of Mollosil in distilled water was $0.59240 \%$, acidic saliva was $1.03820 \%$, basic saliva was $0.33960 \%$, neutral saliva was $0.31920 \%$, and denture cleanser was $0.05100 \%$.

12. After 1, 4 and 16 weeks, Mollosil in denture cleanser showed the least sorption, followed by neutral saliva, basic saliva and distilled water, while the highest sorption was seen acidic saliva; as depicted in Graph 3 .

13. Table 4 shows the mean solubility of Mollosil ; after 1 week, in distilled water was $0.77430 \%$, acidic saliva was $0.96480 \%$, basic saliva was $0.38400 \%$, neutral saliva was $0.30730 \%$, and denture cleanser was 0.05500 $\%$.

14. After 4 weeks, the mean solubility of Mollosil in distilled water was $0.77780 \%$, acidic saliva was $0.97270 \%$, basic saliva was $0.40770 \%$, neutral saliva was $0.33920 \%$ and denture cleanser was $0.05940 \%$.

15. After 16 weeks, the mean solubility of Mollosil in distilled water was $0.78320 \%$, acidic saliva was $0.98860 \%$, basic saliva was $0.42430 \%$, neutral saliva was $0.36940 \%$, and denture cleanser was $0.06360 \%$.

16. After 1, 4 and 16 weeks, Mollosil in denture cleanser showed the least solubility, followed by neutral saliva, basic saliva and distilled water, while the highest solubility was seen in acidic saliva; as depicted in Graph 4

17. Table 5- the mean sorption of Viscogel; after 1 week, in distilled water was $2.772200 \%$,

18. acidic saliva was $2.508900 \%$, basic saliva was $2.319800 \%$, neutral saliva was $2.112600 \%$ and denture cleanser was $1.786300(\%)$.

19. After 4 weeks, the mean sorption of Viscogel in distilled water was 2.923800 (\%), acidic saliva was 2.665900 $\%$, basic saliva was $2.324500(\%)$, neutral saliva was $2.129900 \%$ and denture cleanser was $1.793500 \%$.After 16 weeks, the mean sorption of Viscogel in distilled water was $3.138000 \%$, acidic saliva was $2.879600 \%$, basic saliva was $2.454200 \%$, neutral saliva was $2.182600 \%$ and denture cleanser was $1.801100 \%$.

20. After 1, 4, and 16 weeks, Viscogel in denture cleanser showed the least sorption, followed by neutral saliva, basic saliva, and acidic saliva, while the highest sorption was seen in distilled water; as depicted in Graph 5 .

21. Table 6 shows the mean solubility of Viscogel; after 1 week, in distilled water was $3.102500 \%$, acidic saliva was $3.508500 \%$, basic saliva was $3.167800 \%$, neutral saliva was $3.032600 \%$ and denture cleanser was $2.538000 \%$.

22. After 4 weeks, the mean solubility of Viscogel in distilled water was $3.207200 \%$, acidic saliva was 3.617500 $\%$, basic saliva was $3.172800 \%$, neutral saliva was $2.868600 \%$ and denture cleanser was $2.779300 \%$.

23. After 16 weeks, the mean solubility of Viscogel in distilled water was $3.476800 \%$, acidic saliva was 3.920000 $\%$, basic saliva was $3.328400 \%$, neutral saliva was $2.816500 \%$ and denture cleanser was $2.883100 \%$.

24. After 1, 4 and 16 weeks, Viscogel in denture cleanser showed the least solubility, followed by neutral saliva, basic saliva, distilled water, while the highest solubility was seen in acidic saliva; as depicted in Graph 6 .

25. With the exception of Molloplast-B, all the soft lining material showed higher solubility in artificial saliva than in distilled water. In addition, the percentage absorption of all these materials was lower in artificial saliva than in distilled water.

Table 1:-The mean solubility of values (\%) of Molloplast-B in five different immersing solutions at 1,4 and 16 weeks.

\begin{tabular}{|l|l|l|l|}
\hline \multicolumn{1}{|l|}{ Solubility } & Mean(\%) & Standard deviation (SD) \\
\hline $1^{\text {st }}$ week & Distilled & .79430 & .081586 \\
& Water & & .034820 \\
\cline { 2 - 4 } & $\begin{array}{l}\text { Acidic } \\
\text { Saliva }\end{array}$ & .60480 & \\
\cline { 2 - 4 } & Basic & .38400 & .025932 \\
\hline Saliva & & .030576 \\
\cline { 2 - 4 } & $\begin{array}{l}\text { Neutral } \\
\text { Saliva }\end{array}$ & .30730 & .004397 \\
\cline { 2 - 4 } & Denture & .05500 & \\
\hline
\end{tabular}




\begin{tabular}{|c|c|c|c|}
\hline & Cleanser & & \\
\hline \multirow[t]{5}{*}{$4^{\text {th }}$ week } & $\begin{array}{l}\text { Distilled } \\
\text { Water }\end{array}$ & .83060 & .078846 \\
\hline & $\begin{array}{l}\text { Acidic } \\
\text { Saliva }\end{array}$ & .63420 & .041478 \\
\hline & $\begin{array}{l}\text { Basic } \\
\text { Saliva }\end{array}$ & .40770 & .044880 \\
\hline & $\begin{array}{l}\text { Neutral } \\
\text { Saliva }\end{array}$ & .33920 & .032761 \\
\hline & $\begin{array}{l}\text { Dentue } \\
\text { Cleanser }\end{array}$ & .05940 & .002271 \\
\hline \multirow[t]{5}{*}{$16^{\text {th }}$ week } & $\begin{array}{l}\text { Distilled } \\
\text { Water }\end{array}$ & .86960 & .082011 \\
\hline & $\begin{array}{l}\text { Acidic } \\
\text { Saliva }\end{array}$ & .66070 & .025042 \\
\hline & $\begin{array}{l}\text { Basic } \\
\text { Saliva }\end{array}$ & .41920 & .043019 \\
\hline & $\begin{array}{l}\text { Neutral } \\
\text { Saliva }\end{array}$ & .36080 & .029097 \\
\hline & $\begin{array}{l}\text { Denture } \\
\text { Cleanser }\end{array}$ & .06030 & .002214 \\
\hline
\end{tabular}

Table 2:-The mean sorption values (\%) of Molloplast-B in five different immersing solutions at 1, 4 and 16 weeks.

\begin{tabular}{|c|c|c|c|}
\hline \multicolumn{2}{|l|}{ Sorption } & $\operatorname{Mean}(\%)$ & Standard deviation (SD) \\
\hline \multirow[t]{5}{*}{$1^{\text {st }}$ week } & $\begin{array}{l}\text { Distilled } \\
\text { Water }\end{array}$ & .97400 & .084850 \\
\hline & $\begin{array}{l}\text { Acidic } \\
\text { Saliva }\end{array}$ & .56710 & .028493 \\
\hline & $\begin{array}{l}\text { Basic } \\
\text { Saliva }\end{array}$ & .40610 & .032949 \\
\hline & $\begin{array}{l}\text { Neutral } \\
\text { Saliva }\end{array}$ & .31160 & .041918 \\
\hline & $\begin{array}{l}\text { Denture } \\
\text { Cleanser }\end{array}$ & .04910 & .003510 \\
\hline \multirow[t]{5}{*}{$4^{\text {th }}$ week } & $\begin{array}{l}\text { Distilled } \\
\text { Water }\end{array}$ & 1.05970 & .097693 \\
\hline & $\begin{array}{l}\text { Acidic } \\
\text { Saliva }\end{array}$ & .59600 & .019362 \\
\hline & $\begin{array}{l}\text { Basic } \\
\text { Saliva } \\
\end{array}$ & .43230 & .060775 \\
\hline & $\begin{array}{l}\text { Neutral } \\
\text { Saliva }\end{array}$ & .34650 & .055871 \\
\hline & $\begin{array}{l}\text { Denture } \\
\text { Cleanser }\end{array}$ & .05500 & .004876 \\
\hline \multirow[t]{5}{*}{$16^{\text {th }}$ week } & $\begin{array}{l}\text { Distilled } \\
\text { Water }\end{array}$ & 1.09646 & .094718 \\
\hline & $\begin{array}{l}\text { Acidic } \\
\text { Saliva } \\
\end{array}$ & .62660 & .035463 \\
\hline & $\begin{array}{l}\text { Basic } \\
\text { Saliva }\end{array}$ & .46550 & .052555 \\
\hline & $\begin{array}{l}\text { Neutral } \\
\text { Saliva }\end{array}$ & .38310 & .044854 \\
\hline & $\begin{array}{l}\text { Denture } \\
\text { Cleanser }\end{array}$ & .04580 & .006663 \\
\hline
\end{tabular}


Table 3:-The mean sorption values (\%) of Mollosil in five different immersing solutions at 1,4 and 16 weeks.

\begin{tabular}{|c|c|c|c|}
\hline \multicolumn{2}{|l|}{ Sorption } & $\operatorname{Mean}(\%)$ & Standard deviation (SD) \\
\hline \multirow[t]{5}{*}{$1^{\text {st }}$ week } & $\begin{array}{l}\text { Distilled } \\
\text { Water }\end{array}$ & .52850 & .017148 \\
\hline & $\begin{array}{l}\text { Acidic } \\
\text { Saliva }\end{array}$ & .97730 & .089299 \\
\hline & $\begin{array}{l}\text { Basic } \\
\text { Saliva }\end{array}$ & .32580 & .050655 \\
\hline & $\begin{array}{l}\text { Neutral } \\
\text { Saliva }\end{array}$ & .28940 & .037131 \\
\hline & $\begin{array}{l}\text { Denture } \\
\text { Cleanser }\end{array}$ & .04900 & .009286 \\
\hline \multirow[t]{5}{*}{$4^{\text {th }}$ week } & $\begin{array}{l}\text { Distilled } \\
\text { Water }\end{array}$ & .58850 & .005622 \\
\hline & $\begin{array}{l}\text { Acidic } \\
\text { Saliva }\end{array}$ & 1.01250 & .081168 \\
\hline & $\begin{array}{l}\text { Basic } \\
\text { Saliva } \\
\end{array}$ & .33720 & .049757 \\
\hline & $\begin{array}{l}\text { Neutral } \\
\text { Saliva }\end{array}$ & .31280 & .047269 \\
\hline & $\begin{array}{l}\text { Denture } \\
\text { Cleanser }\end{array}$ & .05000 & .009309 \\
\hline \multirow[t]{5}{*}{$16^{\text {th }}$ week } & $\begin{array}{l}\text { Distilled } \\
\text { Water }\end{array}$ & .59240 & .005420 \\
\hline & $\begin{array}{l}\text { Acidic } \\
\text { Saliva }\end{array}$ & 1.03820 & .081048 \\
\hline & $\begin{array}{l}\text { Basic } \\
\text { Saliva }\end{array}$ & .33960 & .049498 \\
\hline & $\begin{array}{l}\text { Neutral } \\
\text { Saliva }\end{array}$ & .31920 & .047600 \\
\hline & $\begin{array}{l}\text { Denture } \\
\text { Cleanser }\end{array}$ & .05100 & .008433 \\
\hline
\end{tabular}

Table 4:-The mean solubility values (\%) of Mollosil in five different immersing solutions at 1,4 and 16 weeks.

\begin{tabular}{|l|l|l|l|}
\hline \multicolumn{1}{|l|}{ Solubility } & Mean(\%) & Standard deviation (SD) \\
\hline $1^{\text {st }}$ week & $\begin{array}{l}\text { Distilled } \\
\text { Water }\end{array}$ & .77430 & .087683 \\
\cline { 2 - 4 } & $\begin{array}{l}\text { Acidic } \\
\text { Saliva }\end{array}$ & .96480 & .033914 \\
\cline { 2 - 4 } & $\begin{array}{l}\text { Basic } \\
\text { Saliva }\end{array}$ & .38400 & .025932 \\
\cline { 2 - 5 } & $\begin{array}{l}\text { Neutral } \\
\text { Saliva }\end{array}$ & .30730 & .030576 \\
\cline { 2 - 5 } $4^{\text {th }}$ week & $\begin{array}{l}\text { Denture } \\
\text { Cleanser }\end{array}$ & .05500 & .004397 \\
\hline & $\begin{array}{l}\text { Distilled } \\
\text { Water }\end{array}$ & .77780 & .088398 \\
\cline { 2 - 5 } & $\begin{array}{l}\text { Acidic } \\
\text { Saliva }\end{array}$ & .97270 & .034202 \\
\cline { 2 - 5 } & $\begin{array}{l}\text { Basic } \\
\text { Saliva }\end{array}$ & .40770 & .044880 \\
\cline { 2 - 5 } & $\begin{array}{l}\text { Neutral } \\
\text { Saliva }\end{array}$ & .33920 & .032761 \\
\cline { 2 - 4 } & $\begin{array}{l}\text { Denture } \\
\text { Cleanser }\end{array}$ & .05940 & .002271 \\
\hline
\end{tabular}




\begin{tabular}{|c|c|c|c|}
\hline \multirow[t]{5}{*}{$16^{\text {th }}$ week } & $\begin{array}{l}\text { Distilled } \\
\text { Water }\end{array}$ & .78320 & .087809 \\
\hline & $\begin{array}{l}\text { Acidic } \\
\text { Saliva }\end{array}$ & .98860 & .036237 \\
\hline & $\begin{array}{l}\text { Basic } \\
\text { Saliva }\end{array}$ & .42430 & .042557 \\
\hline & $\begin{array}{l}\text { Neutral } \\
\text { Saliva }\end{array}$ & .36940 & .024825 \\
\hline & $\begin{array}{l}\text { Denture } \\
\text { Cleanser }\end{array}$ & .06360 & .002757 \\
\hline
\end{tabular}

Table 5:-The mean sorption values (\%) of Viscogel in five different immersing solutions at 1, 4 and 16 weeks.

\begin{tabular}{|c|c|c|c|}
\hline \multicolumn{2}{|l|}{\begin{tabular}{|l|} 
Sorption \\
\end{tabular}} & $\operatorname{Mean}(\%)$ & Standard deviation (SD) \\
\hline \multirow[t]{5}{*}{$1^{\text {st }}$ week } & $\begin{array}{l}\text { Distilled } \\
\text { Water } \\
\end{array}$ & 2.772200 & .0839839 \\
\hline & $\begin{array}{l}\text { Acidic } \\
\text { Saliva }\end{array}$ & 2.508900 & .0965222 \\
\hline & $\begin{array}{l}\text { Basic } \\
\text { Saliva }\end{array}$ & 2.319800 & .1300972 \\
\hline & $\begin{array}{l}\text { Neutral } \\
\text { Saliva }\end{array}$ & 2.112600 & .0998768 \\
\hline & $\begin{array}{l}\text { Denture } \\
\text { Cleanser }\end{array}$ & 1.786300 & .1001577 \\
\hline \multirow[t]{5}{*}{$4^{\text {th }}$ week } & $\begin{array}{l}\text { Distilled } \\
\text { Water }\end{array}$ & 2.923800 & .0579018 \\
\hline & $\begin{array}{l}\text { Acidic } \\
\text { Saliva }\end{array}$ & 2.665900 & .1093917 \\
\hline & $\begin{array}{l}\text { Basic } \\
\text { Saliva } \\
\end{array}$ & 2.324500 & .1256868 \\
\hline & $\begin{array}{l}\text { Neutral } \\
\text { Saliva }\end{array}$ & 2.129900 & .0905127 \\
\hline & $\begin{array}{l}\text { Denture } \\
\text { Cleanser }\end{array}$ & 1.793500 & .0836185 \\
\hline \multirow[t]{5}{*}{$16^{\text {th }}$ week } & $\begin{array}{l}\text { Distilled } \\
\text { Water } \\
\end{array}$ & 3.138000 & .1354761 \\
\hline & $\begin{array}{l}\text { Acidic } \\
\text { Saliva } \\
\end{array}$ & 2.879600 & .0915062 \\
\hline & $\begin{array}{l}\text { Basic } \\
\text { Saliva }\end{array}$ & 2.454200 & .1347482 \\
\hline & $\begin{array}{l}\text { Neutral } \\
\text { Saliva }\end{array}$ & 2.182600 & .0925157 \\
\hline & $\begin{array}{l}\text { Denture } \\
\text { Cleanser }\end{array}$ & 1.801100 & .2021751 \\
\hline
\end{tabular}

Table 6 :-The mean solubility values (\%) of Viscogel in five different immersing solutions at 1,4 and 16 weeks.

\begin{tabular}{|l|l|l|l|}
\hline Solubility & Mean(\%) & Standard deviation (SD) \\
\hline \multirow{5}{*}{$1^{\text {st }}$ week } & $\begin{array}{l}\text { Distilled } \\
\text { Water }\end{array}$ & 3.102500 & .0625748 \\
\cline { 2 - 4 } & $\begin{array}{l}\text { Acidic } \\
\text { Saliva }\end{array}$ & 3.508500 & .0730909 \\
\cline { 2 - 4 } & $\begin{array}{l}\text { Basic } \\
\text { Saliva }\end{array}$ & 3.167800 & .0562135 \\
\cline { 2 - 4 } & $\begin{array}{l}\text { Neutral } \\
\text { Saliva }\end{array}$ & 3.032600 & .1072579 \\
\cline { 2 - 4 } & Denture & 2.538000 & .1449222 \\
\hline
\end{tabular}




\begin{tabular}{|c|c|c|c|}
\hline & Cleanser & & \\
\hline \multirow{5}{*}{$4^{\text {th }}$ week } & $\begin{array}{l}\text { Distilled } \\
\text { Water }\end{array}$ & 3.207200 & .0808686 \\
\hline & $\begin{array}{l}\text { Acidic } \\
\text { Saliva }\end{array}$ & 3.617500 & .0703914 \\
\hline & $\begin{array}{l}\text { Basic } \\
\text { Saliva }\end{array}$ & 3.172800 & .0570746 \\
\hline & $\begin{array}{l}\text { Neutral } \\
\text { Saliva }\end{array}$ & 2.868600 & .4377526 \\
\hline & $\begin{array}{l}\text { Denture } \\
\text { Cleanser }\end{array}$ & 2.779300 & .2107938 \\
\hline \multirow{5}{*}{$16^{\text {th }}$ week } & $\begin{array}{l}\text { Distilled } \\
\text { Water }\end{array}$ & 3.476800 & .2224319 \\
\hline & $\begin{array}{l}\text { Acidic } \\
\text { Saliva } \\
\end{array}$ & 3.920000 & .0593970 \\
\hline & $\begin{array}{l}\text { Basic } \\
\text { Saliva }\end{array}$ & 3.328400 & .1667874 \\
\hline & $\begin{array}{l}\text { Neutral } \\
\text { Saliva }\end{array}$ & 2.816500 & .1072539 \\
\hline & $\begin{array}{l}\text { Denture } \\
\text { Cleanser }\end{array}$ & 2.883100 & .3327109 \\
\hline
\end{tabular}

Graph 1:-The mean solubility (\%) of Mollolplast-B in five different immersing solutions at 1, 4, and 16 weeks.

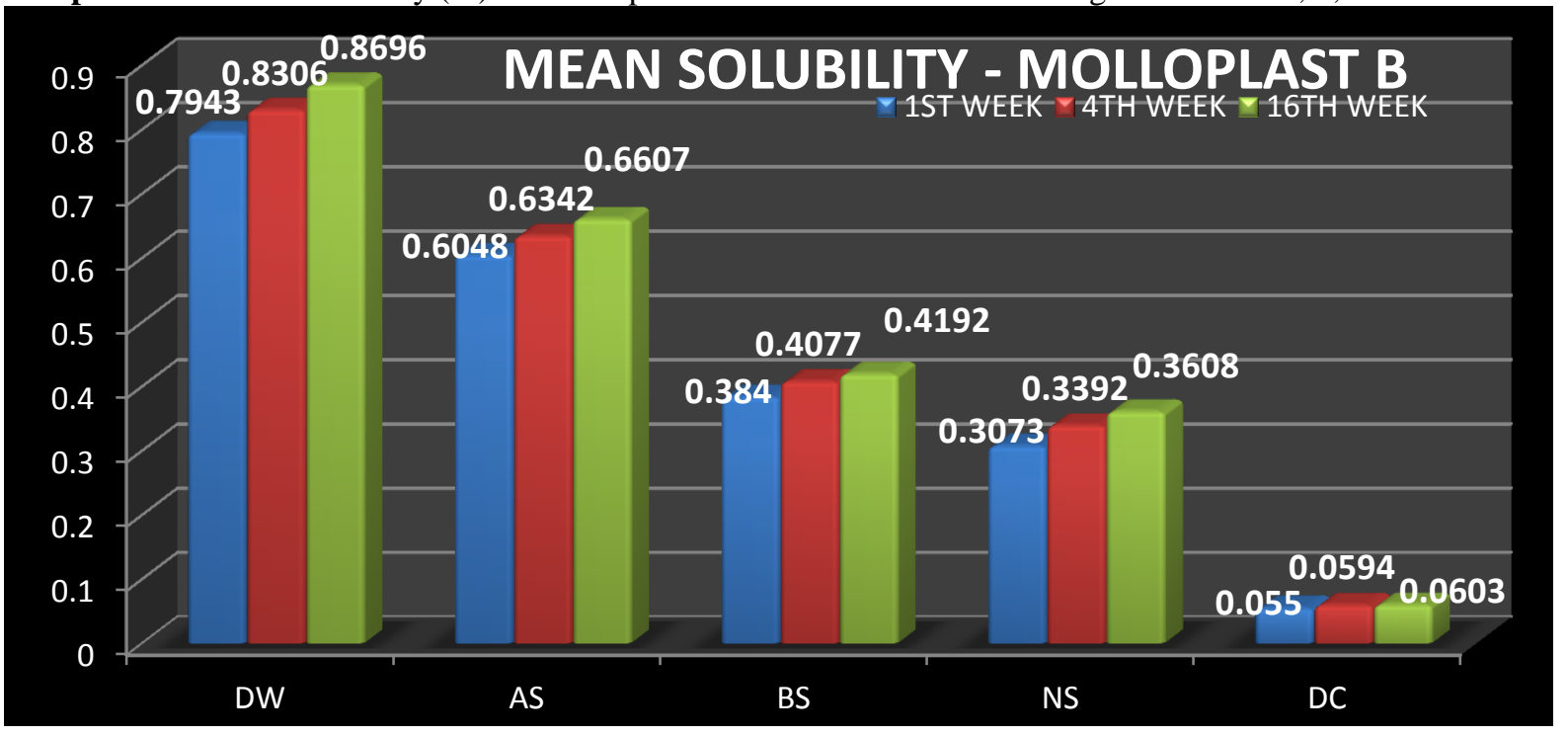


Graph 2:-The mean sorption (\%) of Molloplast-B in five different immersing solutions at 1, 4 and 16 weeks.

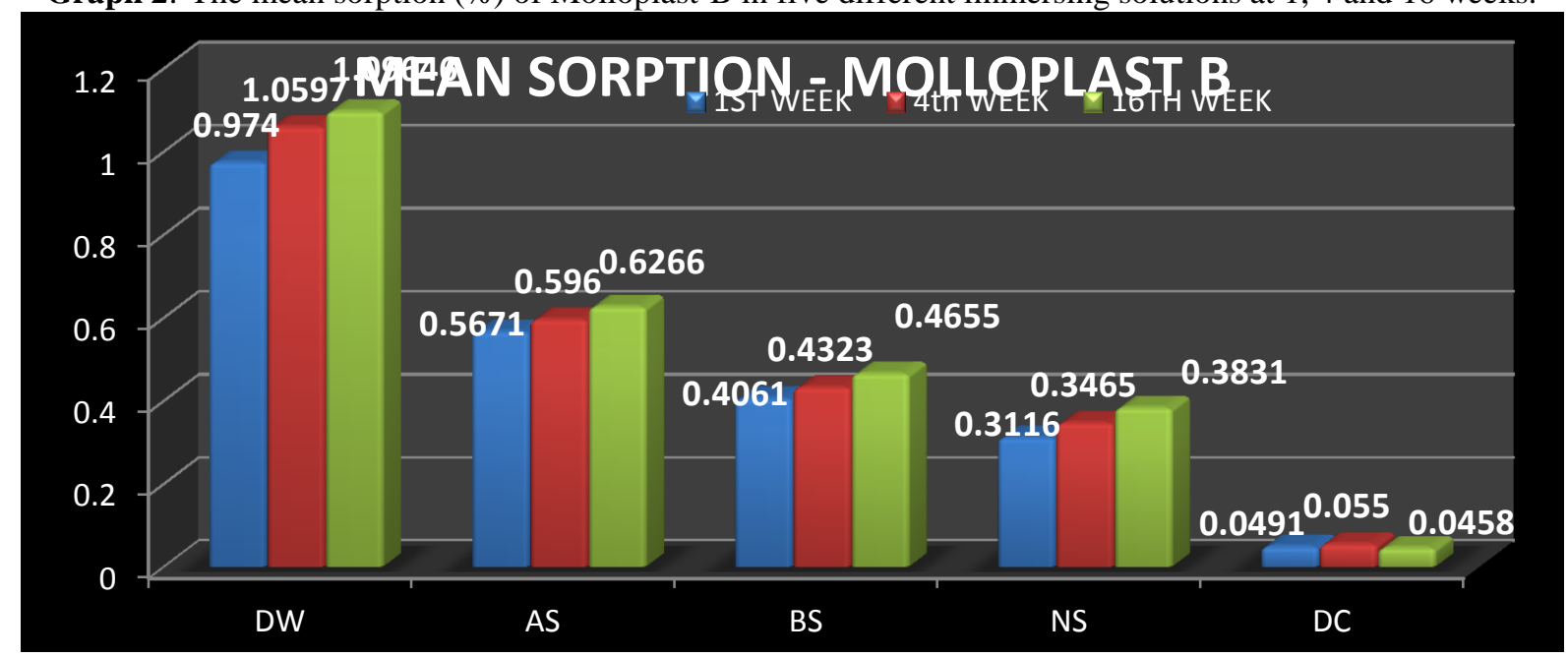

Graph 3:-The mean sorption (\%) of Mollosil in five different immersing solutions at 1, 4 and 16 week.

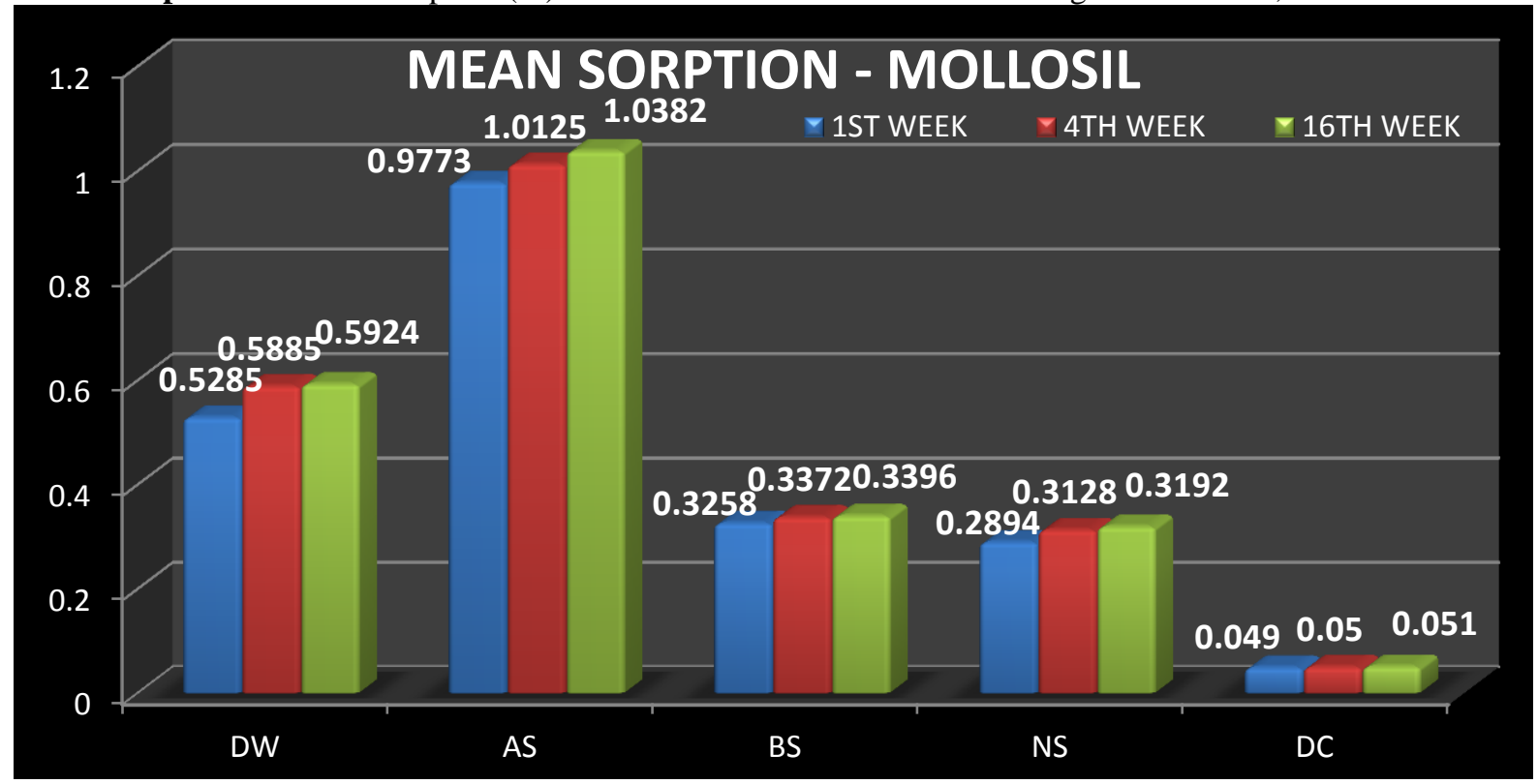


Graph 4:-The mean solubility (\%) of Mollosil in five different immersing solutions at 1,4 and 16 weeks.

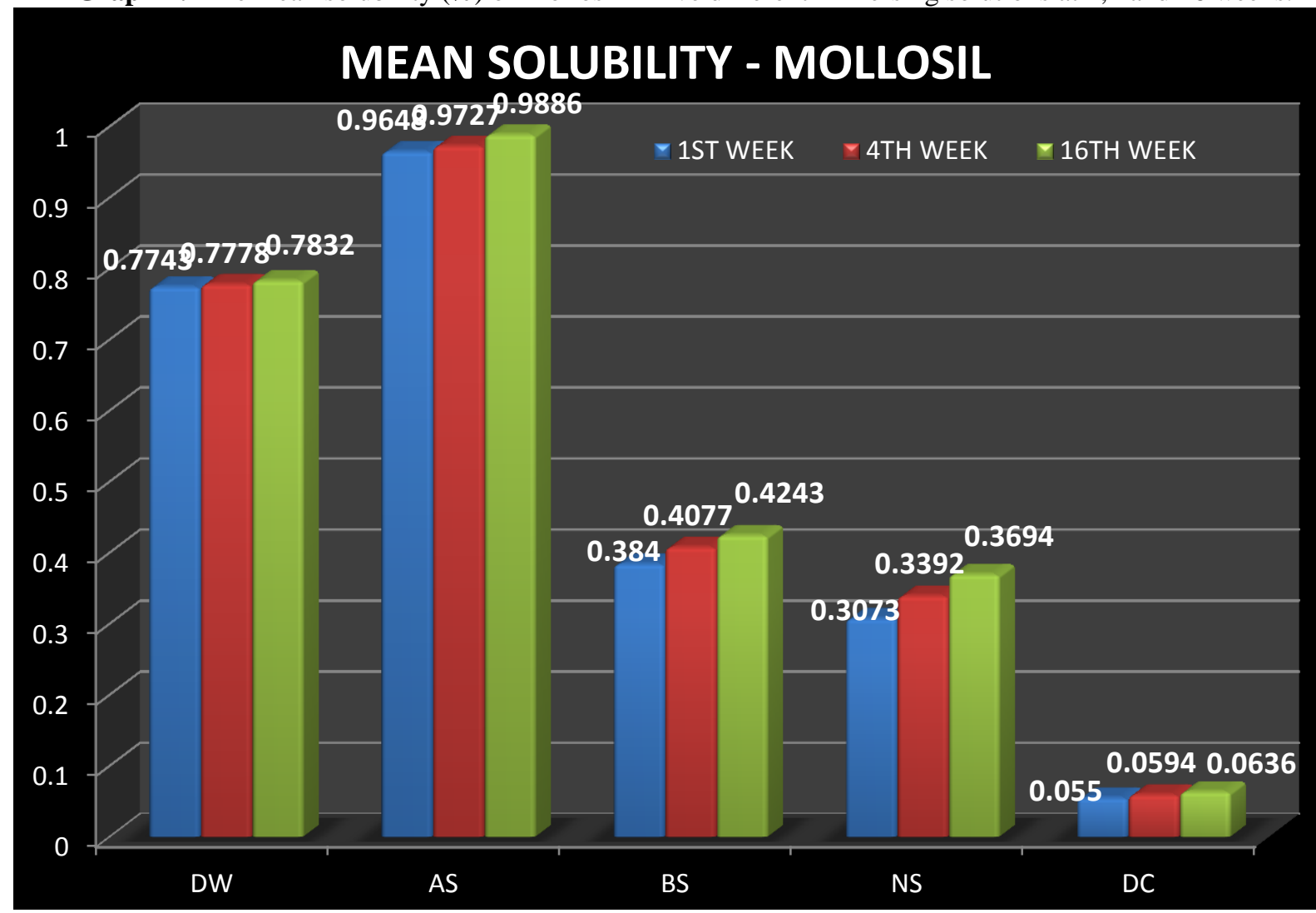

Graph 5:-The mean sorption (\%) of Viscogel in five different immersing solutions at 1, 4 and 16 weeks.

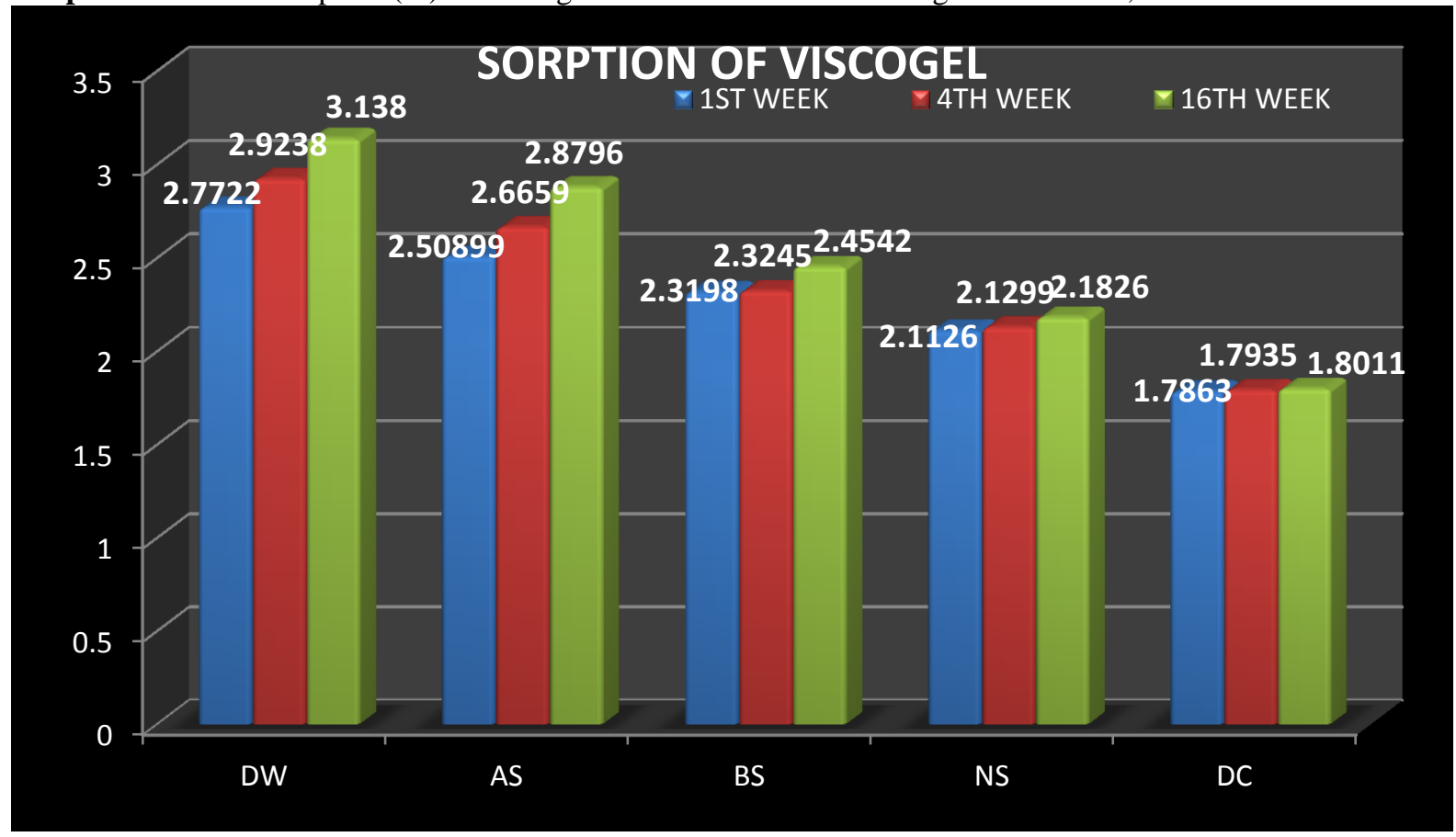


Graph 6 - The mean solubility (\%) of Viscogel in five different immersing solutions at 1,4 and 16 weeks.

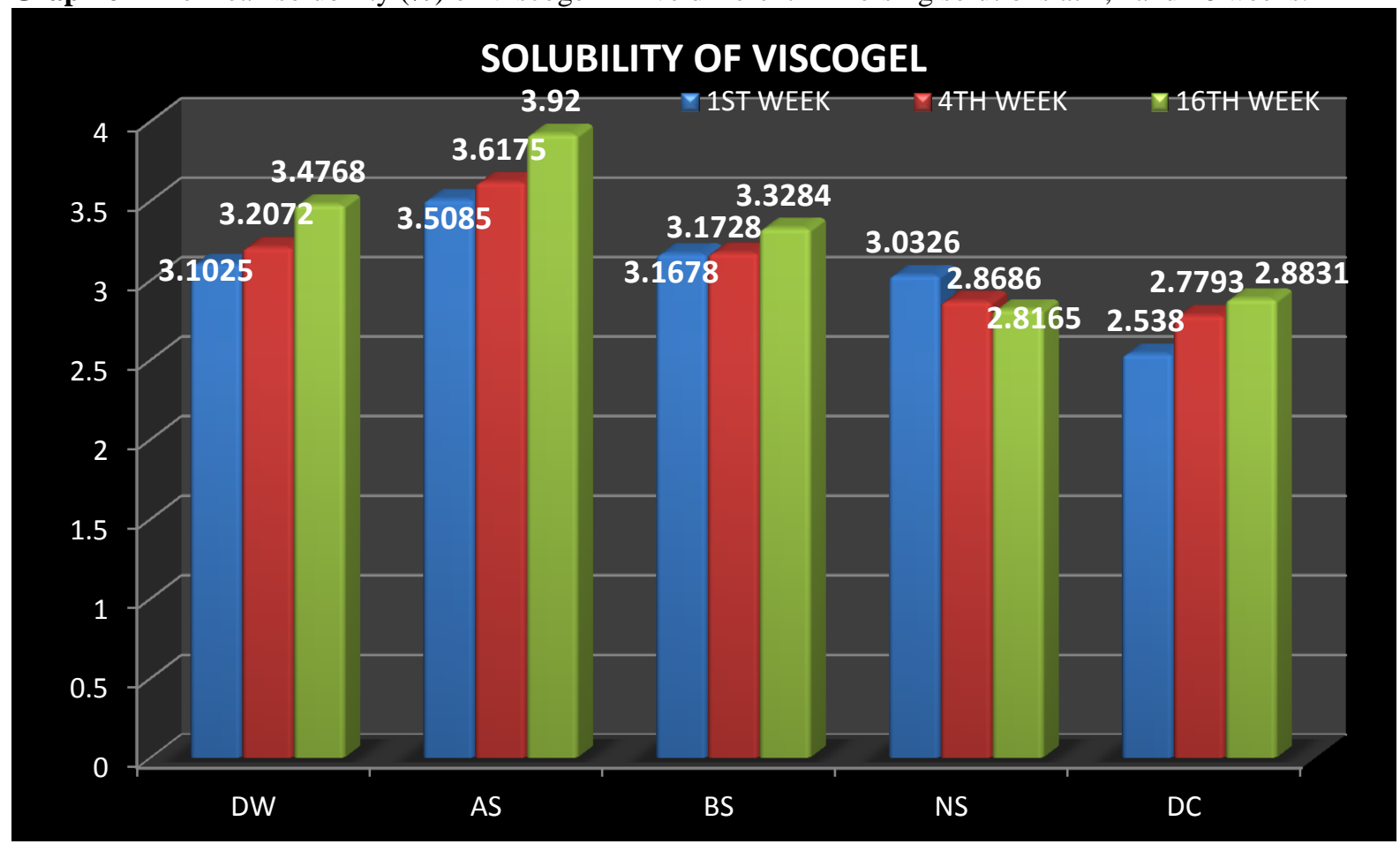

\section{Discussion:-}

The use of soft denture liners is an important adjunct in the treatment of complete and partial denture patients, particularly those who are medically compromised. Water sorption and solubility of soft denture liners are properties that are overlooked in the evaluation of these elastomers. ${ }^{6}$

Soft lining materials undergo two processes when immersed in water. Plasticizers and other soluble materials are leached into the water, and water is absorbed by the polymer. The balance between these two processes affects both the compliance and dimensional stability of the materials. ${ }^{(9)}$ The rate at which the materials absorbed water or lost soluble components varied considerably, with the type of material, the amount of plasticizer or filler content and the solution in which they were immersed. ${ }^{13}$

The study comprised of 3 groups of 50 specimens of each material of which Group 2 was divided into 3 sub groups of each material (Mollosil, Molloplast-B and Viscogel). The groups were divided into artificial saliva (acidic saliva, basic saliva and neutral saliva), distilled water, and denture cleanser and the temperature was set to $37^{\circ} \mathrm{C} \pm 2{ }^{\circ} \mathrm{C}$, to simulate the oral environment. It was established that the percentage solubility of Mollosil and Viscogel in artificial saliva was significantly higher than that in distilled water.

Molloplast -B, a heat cured (HTV), silicone soft lining material, showed higher solubility and sorption in distilled water, and lowest in denture cleanser after 1, 4 and 16 weeks. According to a study conducted by Yanikoglu and Duymus in 2004 it was concluded that, Mollosil and Molloplast-B showed the lowest water sorption values, which was in agreement with the study conducted by Braden and Wright, ${ }^{9}$ but the current study shows that highest water sorption of Molloplast -B in distilled water was not in agreement with the previous studies.

This could be due to a chemical change that is taking place in the polyphosphazene fluoroelastomer contributing to an increase in mass. Low values of water sorption and solubility may be due to improved bonding of the filler to the silicone, which is achieved when the material is cross-linked by heat application. ${ }^{21}$ The highest sorption and solubility of Molloplast-B in distilled water, could be due to the inorganic filler and the correlation to the type of filler and the degree to which the filler is bonded. ${ }^{20}$ 
Chemical cleansers, (denture cleanser- dilute sodium hypochlorite, mild bleach, chemicals such as sodium bicarbonate, which alkalizes water and cleans the denture and citric acid, removes stain), has been considered an efficacious method to prevent C.albicans invasion and denture plaque formation, some types of denture cleansers have been reported to cause significant deterioration of tissue conditioners in a relatively short time. The grade of surface porosity of soft liners varied depending on the immersion time and the combination of denture cleansers and soft liner. ${ }^{1}$

The lower uptake in artificial saliva $\left(\mathrm{NaCl}: 0.400 \mathrm{gm} ; \mathrm{KCl}\right.$ : $0.400 \mathrm{gm} ; \mathrm{CaCl}_{2} \mathrm{H}_{2} \mathrm{O}: 0.795 \mathrm{gm} ; \mathrm{NaH}_{2} \mathrm{PO}_{4}: 0.69 \mathrm{gm}$; $\mathrm{Na}_{2} \mathrm{~S} .9 \mathrm{H}_{2} \mathrm{O}: 0.005 \mathrm{gm}$; Urea $1.0 \mathrm{gm}$; distilled water: $100 \mathrm{ml}$ ) is explicable in terms of the ionic impurities in the polymer. This leads to an enhanced uptake in distilled water since water droplets will form at the impurity sites until elastic and osmotic forces balance. The osmotic pressure will be proportional to the difference in ionic concentrations between the polymer and external liquid, this difference being greater for water than for artificial saliva. For the acrylic resin linings, the absorption of water considerably exceeded the loss of plasticiser and other soluble materials. ${ }^{13}$

Mollosil, auto-polymerizing (RTV), silicone soft liner, showed highest solubility and sorption in acidic saliva, and lowest sorption and solubility in denture cleanser after 1, 4 and 16 weeks. There was a little difference in the percentage solubility in distilled water between the $1^{\text {st }}$ and the $16^{\text {th }}$ week, probably because it does not contain a plasticiser in its composition.

Viscogel, an acrylic based resin soft lining material, showed highest solubility in artificial saliva and highest sorption in distilled water, after 1, 4 and 16 weeks. This is probably due to the loss of ethyl alcohol and the leaching out of lower molecular weight of plasticiser from the set material. The absorption of water by the material results in a weight and volume increase. The observed weight loss probably occurred because, the plasticizers are more soluble in ionic solutions than in water. ${ }^{15}$

With the exception of Molloplast-B, all soft lining materials studied showed higher solubility in artificial saliva than in distilled water. The values obtained in this study is similar when compared with the one observed by Kazanji et al. ${ }^{13}$ High sorption and solubility of soft denture liners are associated with swelling, distortion, hardening, absorption of odors, support of bacteria, colour changes, and debonding of liners from denture bases. ${ }^{6}$

If a soft lining material is to remain effective over an extended period of clinical use, it must remain compliant, i.e., soft and dimensionally stable, whilst remaining bonded to the denture base material. The absorption of water by the material results in an increase in the weight and volume. The compliance of the materials tested is dependent either on the presence of a plasticiser as in the case of the acrylic materials or in the inherent physical properties of the material as in the case of silicone rubbers. ${ }^{13}$ Hence an ideal material should, therefore, have no component which is soluble in saliva or water and should have a low level of absorption. ${ }^{15}$

The limitation of this study is that, it is an in vitro study and hence some variations might be experienced when tested under in vivo conditions owing to fluctuations in the $\mathrm{p} \mathrm{H}$ of saliva, varying concentrations of ions in the saliva, presence of immunoglobulin's and serum markers in the saliva, different occlusal schemes, use of disinfectants and stain removers during denture maintenance. It was a short term study, thus if it's conducted on a long term basis, there will be more clearance in the value and temperature variation could be a reason in the minor changes in the values obtained.

\section{Conclusion:-}

Within the limitations of the study, the following conclusions were drawn:

1. After 1, 4, and 16 weeks, Molloplast-B, a heat-cured (HTV) silicone soft liner, in denture cleanser showed the least solubility followed by neutral saliva, basic saliva, acidic saliva, and the highest solubility and sorption was seen in distilled water.

2. After 1, 4 and 16 weeks, Mollosil, auto-polymerized (RTV) silicone soft liner, in denture cleanser showed the least sorption, followed by neutral saliva, basic saliva and distilled water, while the highest sorption and solubility was seen acidic saliva.

3. After 1, 4, and 16 weeks, Viscogel, an acrylic resin based soft liner, in denture cleanser showed the least sorption, followed by neutral saliva, basic saliva, and acidic saliva, while the highest sorption was seen in distilled water and highest solubility was seen in acidic saliva. 
4. Except Molloplast-B, all the soft liners showed higher solubility in artificial saliva than in distilled water. The percentage absorption of all these materials was lower in artificial saliva than in distilled water.

\section{Summary}

Resorption of the edentulous alveolar ridge leads to the formation of a sharp and thin alveolar crest which is sensitive to the pressure of prosthetic dentures. When the shock absorbing behaviour of the mucosa is diminished, masticatory impact forces are directly transmitted to the underlying tissue. Soft lining materials are able to form an absorbing layer on the part of denture in contact with the oral mucosa and this allows less traumatic occlusal force transmission.

The use of soft lining materials is designed to distribute functional and non functional stresses more evenly because of elastic behaviour.

The most common problems encountered while using soft denture liners are water sorption and solubility. In use, they are constantly bathed in saliva, when out of the mouth, they are usually immersed in either solution of denture cleansers or water storage. During such immersion, soft lining materials undergo 2 responses: Plasticizers and other soluble components are leached out and water or saliva is absorbed.

This study intended to measure the water sorption and solubility of 3 laboratory processed soft denture liners, two silicone resilient lining material (Mollosil, Molloplast-B) and an acrylic based soft lining material (Viscogel) in different environments (Artificial saliva, Denture cleanser and Distilled water) at various intervals (1 week, 4 week and 16 weeks). To standardize the specimens, 50 specimens for each material: Mollosil, Molloplast-B and Viscogel, were prepared using brass dies of dimensions $50 \mathrm{~mm}$ diameter, $0.5 \mathrm{~mm}$ thickness. The samples were then immersed in: Distilled water, Acidic saliva, Basic saliva, Neutral saliva and Denture cleanser.

To obtain acidic, basic and neutral saliva the $\mathrm{pH}$ of artificial saliva was adjusted to 4, 7, 8 with $\mathrm{NaOH}$ or $\mathrm{HCL}$ and the volume was increased to $1 \mathrm{~L}$. The processed sample, 50 each for two silicones soft liner and 50 acrylic based soft liner were then placed in a sealed container each containing different solutions for 1,4 and 16 weeks at $37 \pm 2{ }^{\circ} \mathrm{C}$.

After each time period, the samples were removed from their containers, excess water was removed by blotting with the filter paper, the samples were then weighed using electronic precision balance, capable of measuring 0.001g. This was the weight of the specimen after absorption.

The amount of soluble material lost was measured by placing the specimens back in the desiccator after each sorption cycle and then weighing them at regular intervals until a constant weight was obtained after desiccation. The statistical tools used were, descriptive statistics, one way analysis of variance (One Way ANOVA) and Post hoc Tukey's test.

This is an in vitro study and hence some variations might be experienced when tested under in vivo conditions owing to fluctuations in the $\mathrm{p} \mathrm{H}$ of saliva, varying concentrations of ions in the saliva, presence of immunoglobulin's and serum markers in the saliva. These factors could modify the sorption and solubility during function. It was concluded that, except Molloplast-B, all soft liners showed higher solubility in artificial saliva than in distilled water. The percentage absorption of all these materials was lower in artificial saliva than in distilled water.

\section{References:-}

1. Goll G, Smith DE, Plein JB. The effect of denture cleansers on temporary soft liners. J Prosthet Dent. 1983 Oct 1;50(4):466-72.

2. El-Hadary A, Drummond JL. Comparative study of water sorption, solubility, and tensile bond strength of two soft lining materials. J Prosthet Dent. 2000 Mar 1;83(3):356-61.

3. Dootz ER, Koran A, Craig RG. Physical property comparison of 11 soft denture lining materials as a function of accelerated aging. J Prosthet Dent. 1993 Jan 1;69(1):114-9.

4. Chladek G, Zmudzki J, Kasperski J. Long-term soft denture lining materials. Materials (Basel), 2014;7(8):581642.

5. Anusavice KJ. Phillips' science of dental materials.11 $1^{\text {th }}$ ed. Elsevier Health Sciences; $\quad$ 2003:655-719.

6. Kawano F, Dootz ER, Koran AI, Craig RG. Sorption and solubility of 12 soft denture liners. J Prosthet Dent. 1994 Oct 1;72(4):393-8. 
7. Parker S, Braden M. Soft prosthesis materials based on powdered elastomers. Biomaterials. 1990 Sep 1;11(7):482-90.

8. Kalachandra S, Turner DT. Water sorption of plasticized denture acrylic lining materials. Dent Mater. 1989 May 1;5(3):161-4.

9. Braden M, Wright PS. Water absorption and water solubility of soft lining materials for acrylic dentures. J Dent Res. 1983 Jun;62(6):764-8.

10. Travaglini EA, Gibbons P, Craig RG. Resilient liners for dentures. J Prosthet Dent. 1960 Jul 1;10(4):664-72.

11. Craig RG, Gibbons P. Properties of resilient denture liners. J Am Dent Assoc. 1961 Sep 1;63(3):382-90.

12. Kazanji MNM, Watkinson AC: Soft lining materials: their absorption of and solubility in artificial saliva. $\mathrm{Br}$ Dent J 1988;165:91.

13. Craig RG, Gibbons P. Properties of resilient denture liners. J Am Dent Assoc. 1961 Sep 1;63(3):382-90.

14. Yanikoglu ND, Duymus ZY. Comparative study of water sorption and solubility of soft lining materials in the different solutions. Dental Mater. 2004;23(2):233-9.

15. Jabbal RS, Datta K. Comparative evaluation of water sorption and solubility of two autopolymerizing soft denture liners in distilled water and artificial saliva. Indian J Dent Sci. 2016 Oct 1;8(4):208.

16. Garg A, Shenoy KK. A comparative evaluation of effect on water sorption and solubility of a temporary soft denture liner material when stored either in distilled water, 5.25\% sodium hypochlorite or artificial saliva: An in vitro study. J Indian Prosthodont Soc. 2016 Jan;16(1):53. 